\title{
An Integrated Model Simulation and Empirical Laboratory on Biological Encounter Rates
}

BY SUSANNE MENDEN-DEUER

\section{PURPOSE OF ACTIVITY}

The following summary outlines a combined computer and laboratory exercise conducted in "Quantitative Ecology of Marine Systems," a class I developed at the Shannon Point Marine Center, Western Washington University. The purpose of the laboratory exercise presented here is to familiarize students with the basic variables that drive biological encounter rates: organism speed, size, and abundance. In this inquiry-based exercise, students progress from developing a conceptual model to empirically testing predictions generated by a quantitative model. The learning objectives, beyond the subject matter, include sampling design, quantitative skills, and the association of conceptual and quantitative models.

\section{AUDIENCE}

The target audience is undergraduates. The content can easily be modified to satisfy graduate students, through intensifying the students' interactions with the theoretical and modeling aspects. Non-majors will benefit from this exercise through emphasis on the many biological processes that are driven by encounter rates.

\section{BACKGROUND}

Many biological rates and processes are determined by individual-level interactions or encounters between organisms and their biotic or abiotic environments. Even abiotic processes, such as chemical reactions and asteroid collisions, are encounter-rate dependent. All of these seemingly disparate processes can be understood within a single framework that considers three variables: organism abundance, size (e.g., organism diameter or perception distance), and motility. The interplay among these three variables provides a quantitative predictor of organism encounter rates with, amongst others, suitable mates (sexual reproduction), suitable prey (predation), and suitable habitat (colonization). Understanding biological encounter rates is therefore fundamentally important to understanding a wide range of ecological phenomena that affect oceanographic rates and processes.

\section{RESEARCH QUESTIONS}

How does organism encounter rate vary with varying abundance, motility, and size? Which biological processes are encounter-rate dependent? Which are the important variables in these processes?

\section{APPROACH}

Students will have some intuition how the aforementioned variables will affect biological encounter rates. The approach taken in this laboratory is to allow students to explore and build upon their intuition. This laboratory consists of two discrete sections: (1) an interactive computer exercise in which students generate hypotheses about factors that could affect encounter rates and (2) a laboratory exercise that tests some of these hypotheses. To allow students to draw on their own intuition, I intentionally do not precede this exercise with a lecture. I offer students Gerritsen and Strickler (1977) for background.

The model simulates random organism movements in two dimensions and keeps track of each organism's encounters with indestructible targets (i.e., targets remain available after they are encountered). Students record the en-

Susanne Menden-Deuer (smd@eno. princeton.edu) is Lecturer, Shannon Point Marine Center, Western Washington University, Anacortes, WA, USA and is currently Research Fellow, Department of Ecology and Evolutionary Biology, Princeton University, Princeton, NJ, USA. 
counter rates given a set of initial values and then, using a graphical interface (Figure 1), modify those values to identify the quantitative significance of each variable. In the process, the class discusses the important steps in formulating and testing a model. Based on the model predictions, students design and conduct an empirical experiment in which they test hypotheses that they generated with the aid of the model simulation (e.g., encounter rate increases with increasing target concentration). Finally, students return to the computer model to examine whether they can reproduce their laboratory observations in silico.

\section{MECHANICS}

Each of the two segments can take between 1-3 hours, depending on how intensely the material is discussed and how much students are guided in their exploration. To decrease the total time required, the computer exercise can be replaced by a guided discussion, or homework exercise that asks students to speculate on the important factors driving encounter rates. The data analysis will be done as an independent or homework exercise.

For the model simulation, students are provided with computer code (to download the model simulation code, go to http://www.tos.org/hands-on/index. html) that displays the model variables in an editable user interface (Figure 1). Upon pressing the "Run Model" button, the simulation displays the positions of targets and searchers in a two-dimensional arena (Figure 2). The time elapsed is displayed on the top of the screen. At the end of the simulation, the average encounter rate is displayed on the screen. If the encounter rate was greater than zero, a second graph shows the variations in encounter rate over time. A series of questions (see below under "Activity") guides students to modify the variables and asks questions that relate the simulation to biological processes.

The empirical test can be done with a number of different organisms (see "Possible Modifications" below). For the laboratory exercise, I used the green urchin (Strongylocentrotus droebachiensis) and injected potassium chloride into the body cavity of 3 to 5 urchins $30 \mathrm{~min}$ utes prior to the lab so students find an ample supply of sperm and eggs (Strathmann, 1987; see Internet link below for detailed instructions on the spawning and fertilization procedure ${ }^{1}$ ). I chose the sea urchin model, because students in my class were already familiar with the fertilization protocol from a prior class, so they could focus on the encounterrate question without being distracted by the mechanics of fertilization (which are simple, however). Furthermore, scoring fertilization success is rapid and unambiguous and allows students to assess large sample sizes and many replicates.

\section{POSSIBLE MODIFICATIONS}

The materials required can be modified depending on the availability of facilities and equipment. If computers are not available for each student to run their own simulations, the model component can be replaced by thought experiments, a guided discussion, or instructor's demonstration of the simulations. The important aspect is that students are asked to predict effects of different variables and changes in their magnitude. The model simulation is only one way to achieve that goal. The use of the computer software will take additional introduction, if students are unfamiliar with computer simulations. The insights to be gained from this exercise are not reliant on the model simulation.

For the empirical test, any experiment that allows students to vary speed (motility, mixing, filtration rate), concentration, or target size will allow suitable tests of the student-generated hypotheses. I have conducted the same laboratory using predator-prey interactions between heterotrophic protists or mussel and phytoplankton prey, where prey concentration is varied and ingestion is measured as the difference in Chl $a$ fluorescence.

This laboratory makes several implicit assumptions in order to minimize the material students need in preparation. For example, here I only refer to "target size" as a variable. In fact, from a theoretical point of view, target size and a searchers' perception distance (e.g., chemosensory, mechanosensory) are identical. Thus, advanced students might delight in exploring how organisms can advertise or conceal themselves through modification of either of these two variables. Similarly, organism movements are simulated as random walks. Real organisms have an enormous repertoire of movement behaviors that increase the probability of desired encounters. A homework assignment to explore such strategies may be a worthwhile exercise. I dedicate a following lab to observing the swimming motions of different types of plankton.

\footnotetext{
The protocol for the sea urchin fertilization is provided at: http://raven.zoology.washington.edu/celldynamics/downloads/urchinlab.html (last accessed September 2006).
} 


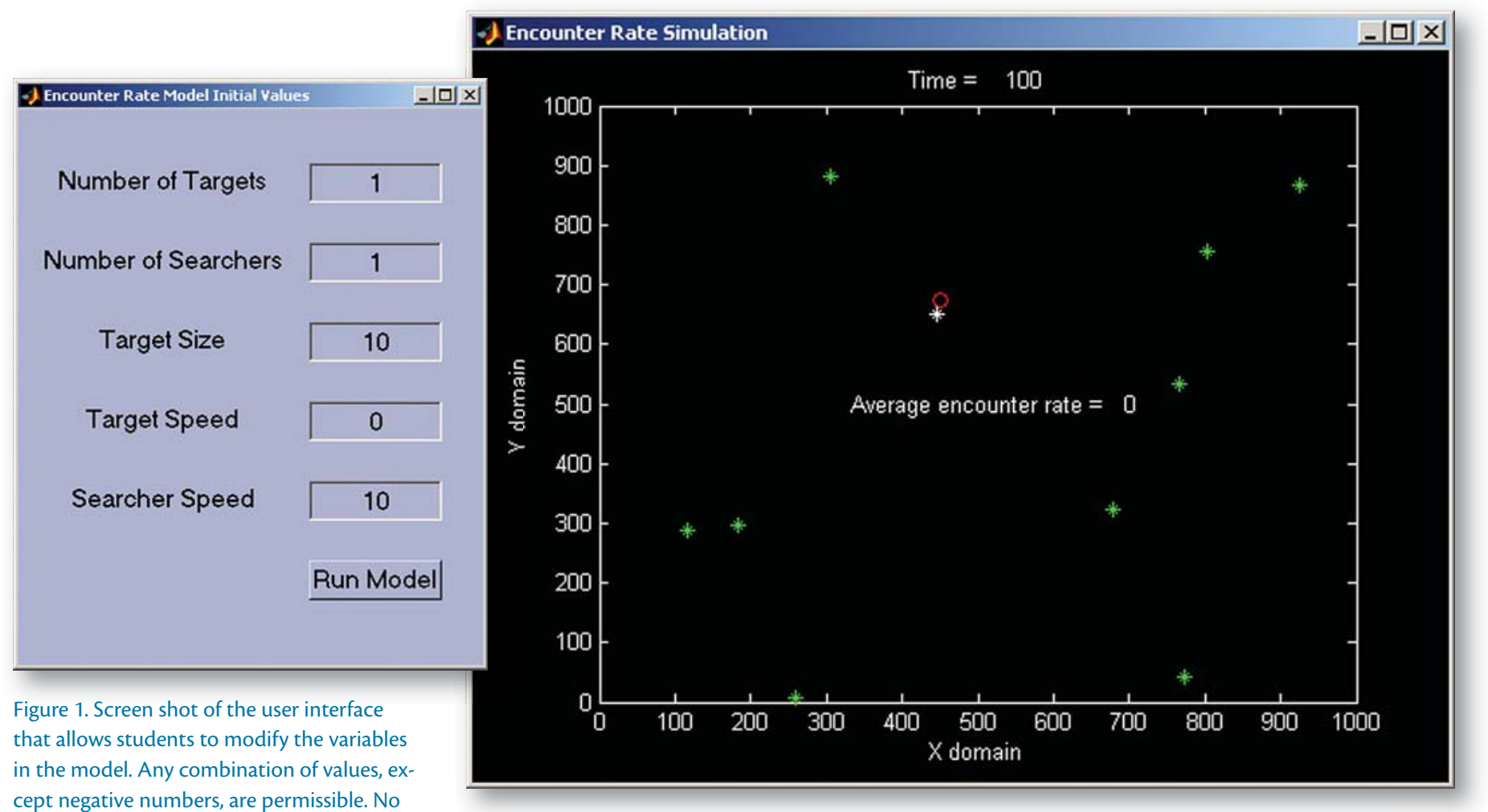

units are given, to allow students to relate

the simulation to a variety of organisms.

Figure 2. Screen shot of a model simulation showing targets (green stars) and searcher (red circles) in a two-dimensional arena. Encountered targets change color to white. At the end of the simulation, the average encounter rate (per time step and searcher) is displayed. The length units for the sea urchin example are microns (i.e., the shown area is $1 \mathrm{~mm}^{2}$ ); each model time step equals 3 seconds. Dimensions of length and time are relative to the particular system modeled. During the lab, students are asked to identify the spatial and temporal units of their model system.

\section{ACTIVITY}

The written instructions given to students are as follows (go to www.tos.org/ hands-on to download an MS Word file of these instructions):

\section{Computer Simulations}

You should treat the computer simulations just like any other experiment. That is, you should make ample notes on your set-up and results. You will be asked to write a laboratory report about both segments of the lab. In your discussion, include answers to at least some of the questions stated below.

1. Measure encounter rates given the model-supplied values. Make sure that

the two files "encounter.m" and "enc_ code.m" are in the same folder. From within Matlab open and execute the code: "encounter.m." A user interface will pop up with some preset values (Figure 1). (Should you ever accidentally "lose" the interface, just run "encounter.m" again.) Click on the "Run Model" button. What happens? What do you see? If your simulation results in an encounter rate, a second graph appears. What does it show? Keep in mind that your results might differ from your fellow students' because there is a considerable random element in each model simulation. It will be useful to keep track of the group results. Close all open graphs and return to the user interface.

2. In sequence, vary each of the following variables: concentration of searchers and targets, target size, swimming speed of searcher and target. What is the biological meaning of each change you made: are the changes realistic? Should you only use realistic values? Keep track of the effects these variations have on changes in encounter rate. For some variables, small changes in values will change encounter rates much more than large changes in other values. Why is that? Note that changes in target size will not be visible. 
3. Based on your model variations, develop a set of predictions specifying the relative importance of each variable for organism encounter rates.

4. Develop an experimental protocol to test those predictions using sea urchin sperm and eggs. Which variables can you change? What should you measure? How? What sample sizes should you gather? Note that the model simulates a hypothetical case and does not provide you with units for space and time dimensions. What will the dimensions of your model system be?

5. For your write-up, think about the model assumptions. What are some shortcomings of this simulation? (For example, targets remain available after they are encountered, feeding or fertilization would 'remove' the target from the pool.) Think of biological manifestations of your model manipulations: how can organisms change their size or speed? What strategies could organisms use to conceal themselves or advertise their presence?

\section{Laboratory Exercise}

Because students are supposed to develop the protocol, I do not supply them with written instructions. I let each student develop a protocol, which we discuss prior to the experiment. The methods suggested here will result in rough estimates of the necessary data. Students are typically very good at recognizing

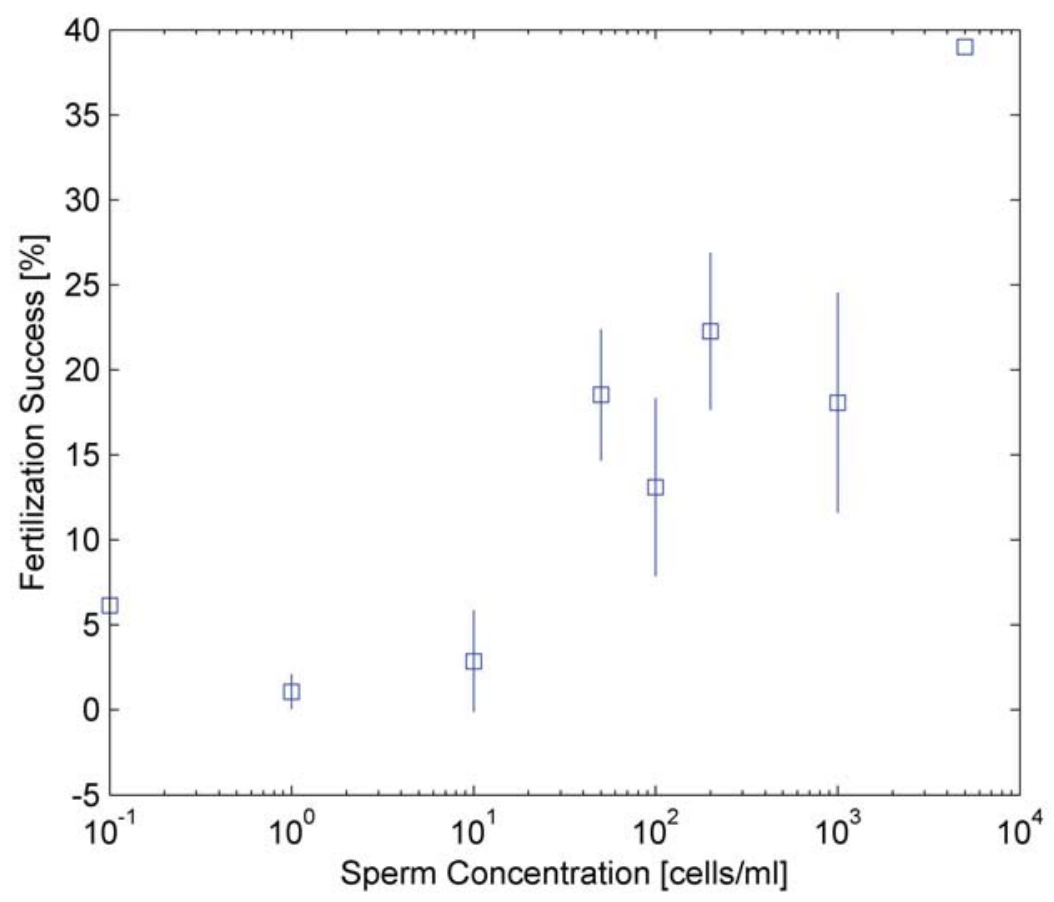

Figure 3. Students' results from triplicate measurements of fertilization success using sperm at six different target concentrations, ranging in dilution from 0.02 to $20 \%$ of the original sperm extract. Maximum fertilization rate in pure sperm was low at $39 \%$. Error bars shown are $+/$ - one standard deviation.

limitations in their methodology. Often, they will suggest very elaborate fixes (e.g., need three-dimensional, high-speed video to measure swimming speeds and a flow cytometer to measure cell-cyclespecific organism densities). I use these discussions to ask students to incorporate logistic constraints in their modified procedures. Together, all students then develop one common protocol. I particularly stress bench skills, sample size, and labeling. As a result of the computer exercise or group discussion, students will have arrived at the conclusion that size, speed, and concentration are all important variables. They typically recognize that they can only alter concentration and that organism swimming speed and size are fixed variables that need to be measured. Using microscope slides (depression or clay-feet raised), students measure size and swimming speeds of sperm and egg (I alert students to the fact that unless they know eggs are non-motile, they need to verify it). Both sperm and eggs are highly concentrated and can be counted with a hemocytometer. With students that had worked with hemocytometers before, this process took about 30 minutes. Then students prepare a 0.02 to 20 percent dilution series of the sperm. The number of dilutions depends on the number of students. I ask each student to conduct at least three fertilization replicates (e.g., three independent trials exposing eggs to a specific concentration of sperm). Using a microscope, students score percent fertilization on 100 eggs per experiment. Provided eggs are dense, scoring of 100 eggs takes less than 10 minutes. With a sample size of at least 12 independent fertilizations, one should see some discussable results (Figure 3 ). 


\section{Assessment}

Students are assessed based on their completion of a homework or laboratory report that emphasizes reporting the data in appropriate written and graphical format, linking the observations and results from the computer and empirical lab and applying the learned material by identifying the model/experimental parameters in other biological processes that are affected by individual-level encounters.

\section{RELATED TOPICS}

Because biological encounter rates are so fundamental to widespread phenomena, I use this exercise early on in my class and frequently refer back to it. Completing this laboratory early also allows me to address some of the important aspects that are not adequately dealt with (e.g., movement behaviors, see "Possible Modifications").

Based on this exercise, students may be interested in exploring the following topics further: predator-prey dynamics and functional response curves; random walks and foraging behaviors; chemical and mechanical signaling; larval settlement; sexual reproduction; encounter probabilities as a consequences of life in a three- (pelagic) or two- (benthic) dimensional habitat; feeding strategies employed by different predators; bacterial colonization of marine snow; sizing and placement of marine reserves; and the effects of turbulence on encounter rate.

This laboratory also provides a wonderful opportunity to introduce students to the value of exploiting theory and model simulations to generate testable hypotheses. Students will benefit from a discussion of the differences and similarities between theoretical and empirical work. It will be particularly useful to help students understand the need to verify assumptions and to discover why models are simplified characterizations of complex processes.

\section{ACKNOWLEDGEMENTS}

I thank Brian Bingham for introducing me to the sea urchin fertilization protocol and the ESCI 497 students for allowing me to test new laboratories with them. Tansy Clay improved an earlier version of this manuscript, as did the constructive comments by Pete Jumars and two anonymous reviewers. I appre- ciate funding from Shannon Point Marine Center to develop this laboratory as well as support from the Deutsche Forschungsgemeinschaft (German National Science Foundation).

\section{REFERENCES}

Gerritsen, J., and J.R. Strickler. 1977. Encounter probabilities and community structure in zooplankton: A mathematical model. Journal of the Fisheries Research Board of Canada 34:73-82.

Strathmann, M.F. 1987. Reproduction and Development of Marine Invertebrates of the Northern Pacific Coast, University of Washington Press, Seattle and London.

\section{FURTHER READING}

Baskett, M.L., M. Yoklavich, and M.S. Love. 2006. Predation, competition, and the recovery of overexploited fish stocks in marine reserves. $\mathrm{Ca}$ nadian Journal of Fisheries and Aquatic Sciences 63:1,214-1,229.

Levitan, D.R., M.A. Sewell, and F.S. Chia. 1991. Kinetics of fertilization in the sea urchin Strongylocentrotus franciscanus: Interaction of gamete dilution, age, and contact time. Biological Bulletin 181:371-378.

Metcalf, A.M., T.J. Pedley, and T.F. Thingstad. 2004. Incorporating turbulence into a plankton foodweb model. Journal of Marine Systems 49:105-122.

Visser, A.W., and T. Kiørboe. 2006. Plankton motility patterns and encounter rates. Oecologia 148:538-546.

\section{HANDS-ON OCEANOGRAPHY}

\section{Visit www.tos.org/hands-on to download activities or submit an activity of your own for consideration.}

Hands-On Oceanography provides peer-reviewed activities appropriate for undergraduate and/or graduate classes in oceanography. Hands-on is broadly interpreted as those activities that actively engage students (i.e., activities where students have to make decisions, record results, and interpret results). Hands-on activities include, but are not limited to, computer-based models and laboratory demonstrations.

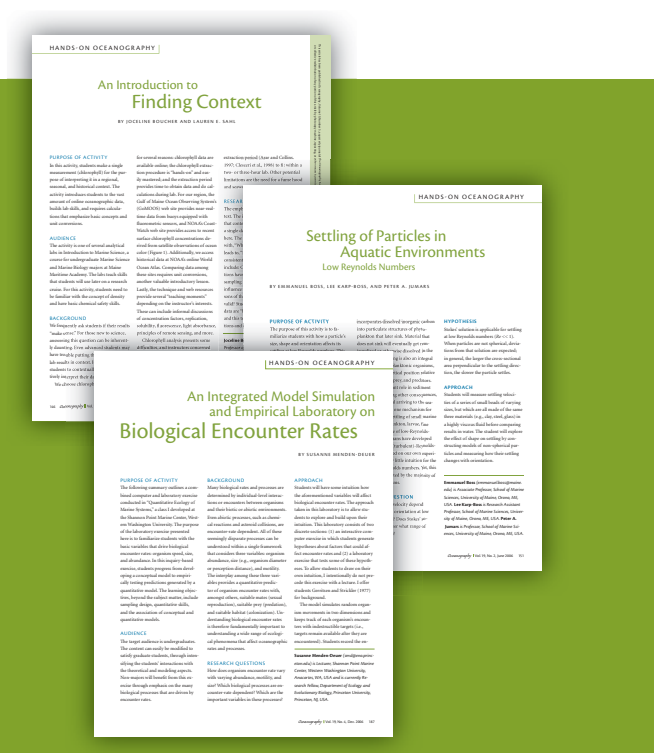

